\title{
Reactive oxygen species and aldehyde dehydrogenase activity in Hodgkin lymphoma cells
}

\author{
Jun-ichiro Ikeda ${ }^{1}$, Suhana Mamat ${ }^{1,2}$, Tian Tian ${ }^{1}$, Yi Wang ${ }^{1}$, Wenjuan Luo ${ }^{1,3}$, Nur Rahadiani ${ }^{1}$, Katsuyuki Aozasa ${ }^{1}$ \\ and Eiichi Morii ${ }^{1}$
}

Tumor cells with tumorigenic potential might be limited to a small population of cells, called cancer-initiating cells (CICs). $\mathrm{CICs}$ efficiently form colonies in vitro, yield both $\mathrm{CIC}$ and non-CIC populations, maintain reactive oxygen species (ROS) at low levels, show high aldehyde dehydrogenase (ALDH) activity, and are mostly in a quiescent state of the cell cycle. CICs of Hodgkin lymphoma (HL) are small in size, with low levels of ROS. The relationship between ROS level and ALDH activity in $\mathrm{CICs}$ was examined in $\mathrm{HL}$ cell lines. ROS-low and ALDH-high populations formed colonies in semi-solid cultures more efficiently than ROS-high and ALDH-low populations. ALDH-high populations yielded both ALDH-low and -high populations, whereas ALDH-low populations rarely yielded an ALDH-high population. The number of cells in a quiescent state was significantly greater in ROS-low than in ROS-high cells, whereas that of ALDH-high and ALDH-low cells was comparable to each other. These findings show that ALDH-high and ROS-low cells share CIC-like potential, but they differ in their cell cycle status, suggesting that CICs are comprised of cells with heterogeneous characteristics. Laboratory Investigation (2012) 92, 606-614; doi:10.1038/labinvest.2012.4; published online 20 February 2012

KEYWORDS: aldehyde dehydrogenase; cancer initiating cells; hodgkin lymphoma; reactive oxygen species; tumorigenicity

Tumors consist of heterogeneous cell populations derived from a single clone. Recently, it has been demonstrated that tumor cells with tumorigenic potential are limited to a small population of cells, called cancer-initiating cells (CICs). CICs are present in several tumors, such as leukemia, breast, brain, and colon cancers. ${ }^{1-8}$ CICs yield both CICs and non-CICs, whereas non-CICs rarely yield CICs. ${ }^{1,5}$ CICs efficiently efflux anti-tumor chemicals, form colonies in semi-solid culture, and degrade reactive oxygen species (ROS) that are generated upon radiation-induced apoptosis. ${ }^{1-9}$

Recent studies have pointed to high aldehyde dehydrogenase (ALDH) activity as a candidate CIC characteristic. ${ }^{10-20}$ ALDH oxidizes retinol to retinoic acid in the early stages of stem cell differentiation. ${ }^{11}$ High ALDH activity is detected in both physiological stem cells, such as hematopoietic and neural stem cells, ${ }^{21,22}$ and CICs of human multiple myeloma, acute myeloid leukemia, and cancers of the lung and breast, ${ }^{10,12-17}$ indicating that ALDH activity may be a common marker for both normal and malignant stem cell populations. To date, information on ALDH activity in CICs of malignant lymphomas is extremely limited.
The cellular origin of Hodgkin lymphoma (HL) had been controversial; however, recent molecular biology studies have revealed that HL is mostly a neoplasia of B lymphocytes. ${ }^{23,24}$ HL can be diagnosed by the presence of multinucleated ReedSternberg (RS) cells and mononuclear Hodgkin cells intermingled with small lymphocytes and various inflammatory cells. They are regarded as pathognomonic for HL and share the expression of some cell markers with Hodgkin cells, such as CD15 and CD30, although the proliferative potential of RS cells was reported to be lower than that of Hodgkin cells. ${ }^{25} \mathrm{To}$ date, markers that can differentiate Hodgkin cells from RS cells have not been established.

We recently reported that HL cell lines, L1236 and L428, were composed of two types of cells: cells with a single nucleus (S) that resemble Hodgkin cells and those with multiple nuclei (M) that resemble RS cells. ${ }^{26} \mathrm{~S}$ cell cultures yielded both $\mathrm{S}$ and $\mathrm{M}$ cells, whereas $\mathrm{M}$ cell cultures yielded only $\mathrm{M}$ cells. When cultured in methylcellulose or inoculated into NOD/SCID mice, colony numbers and tumor sizes were larger for $S$ than for $M$ cells. The concentration of intracellular ROS was low in a portion of $S$ cells that

\footnotetext{
${ }^{1}$ Departments of Pathology, Osaka University Graduate School of Medicine, Yamada-oka, Suita, Japan; ${ }^{2}$ Department of Basic Health Science, Kulliyyah of Allied Health Sciences, International Islamic University Malaysia, Kuantan, Pahang, Malaysia and ${ }^{3}$ Research and Engineering Center for Natural Medicine, School of Medicine, Xi'an JiaoTong University, Xi'an, Shaanxi Province, PR China

Correspondence: Dr E Morii, Department of Pathology, Graduate School of Medicine, Osaka University, Yamada-oka 2-2, Suita 565-0871, Japan.

E-mail: morii@patho.med.osaka-u.ac.jp

Received 2 September 2011; revised 23 November 2011; accepted 24 November 2011
} 
abundantly expressed FoxO3a, a transcription factor regulating ROS-degrading enzymes. These ROS-low $\mathrm{S}$ cells were tumorigenic and possessed CIC-like characteristics.

Jones $e t a l^{27}$ reported that cell lines and circulating B cells from HL patients contained a limited number of clonal ALDH-high B cells that might initiate HL. However, a correlation between ROS levels and ALDH activity has not yet been determined in any type of tumor, including HL. Here, ALDH activity was examined in HL cell lines with regard to tumorigenic potential. Although both high ALDH activity and low ROS levels may be candidate CIC markers, the present study revealed that the cell cycle status among ALDH-high and ROS-low cells varied, suggesting heterogeneity of CIC-like populations.

\section{MATERIALS AND METHODS Cell Lines}

Hodgkin lymphoma cell lines, L428 and L1236, were purchased from Deutsche Sammlung von Mikroorganismen and Zellkulturen (Braunschweig, Germany). Cells were cultured in RPMI 1640 (Sigma, St Louis, MO, USA) supplemented with $10 \%$ fetal calf serum (FCS; Nippon BioSupplyCenter, Tokyo, Japan). A portion of cultured cells was cytospun on poly-L-lysine-coated glass slides at $1500 \mathrm{rpm}$ for $3 \mathrm{~min}$ and stained with Giemsa solution. As previously reported, ${ }^{26}$ cells with a single nucleus or a multinucleus (more than two nuclei) were named $\mathrm{S}$ and $\mathrm{M}$ cells, respectively.

\section{Isolation of ALDH-Low and ALDH-High Cells}

To isolate the cell population with high ALDH enzymatic activity, the Aldefluor kit (Stem Cell Technologies, Vancouver, BC, Canada) was used, according to the manufacturer's instructions. Briefly, cells were suspended in Aldefluor assay buffer containing ALDH substrate and BODIPY-aminoacetaldehyde (BAAA). The BAAA was taken up by living cells and converted by intracellular ALDH into BODIPY-aminoacetate, which yields bright fluorescence. The brightly fluorescent ALDHexpressing cells were detected with a FACS Calibur or FACS Aria II (BD Biosciences, Franklin Lakes, NJ, USA). As a negative control, cells were stained under identical conditions with the specific ALDH inhibitor diethylaminobenzaldehyde (DEAB; Sigma, St Louis, MO, USA). Data were analyzed by Cell Quest software (BD Biosciences). In HL cell lines, cells with bright and no or faint fluorescence were defined as ALDH-high and ALDH-low, respectively.

\section{In Vitro Colony Formation Assay}

A total of 5000 suspended cells were plated in culture dishes with $1 \mathrm{ml}$ methylcellulose-containing Dulbecco's Modified Eagle's Medium (DMEM) supplemented with 15\% FCS. The number of colonies was counted on day 14 .

\section{Assay for Intracellular ROS Level}

Cells were incubated with $10 \mu \mathrm{M}$ of 5-(and-6)-chloromethyl$2^{\prime}, 7^{\prime}$-dichlorodihydrofluorescein diacetate, acetyl ester
(CM- $\mathrm{H}_{2}$ DCFDA, Invitrogen) at $37^{\circ} \mathrm{C}$ for $20 \mathrm{~min}$. Dead cells were stained with propidium iodide (PI). ROS-high cells were stained in green and dead cells in red. At least 200 of $\mathrm{S}$ and $\mathrm{M}$ cells, respectively, were examined, and the proportion of ROS-high viable cells was counted.

\section{Isolation of ROS-Low and ROS-High Cells}

To isolate the cell population with high ROS activity, cells were incubated with $10 \mu \mathrm{M}$ CM- $\mathrm{H}_{2} \mathrm{DCFDA}$ at $37^{\circ} \mathrm{C}$ for $20 \mathrm{~min}$. Dead cells were stained with PI. Brightly fluorescent cells were detected with a FACS Calibur or FACS Aria II. As a negative control, cells were left unstained under identical conditions. Data were analyzed using Cell Quest software. In HL cell lines, cells with bright and no or faint fluorescence were defined as ROS-high and ROS-low, respectively.

\section{Flow Cytometry and Real-Time Quantitative Reverse Transcription-Polymerase Chain Reaction (qRT-PCR)}

Cells were stained with $\mathrm{CM}-\mathrm{H}_{2}$ DCFDA, and ROS-high and -low cells were sorted with a FACSAria II. Total RNA was extracted from sorted cells using the RNeasy RNA extraction kit (Qiagen, Valencia, CA, USA), according to the manufacturer's protocol. cDNA was synthesized using oligo (dT) primers and SuperScript III reverse transcriptase (Invitrogen). qRT-PCR was performed with an ABI PRISM 7700 instrument (Applied Biosystems, Foster City, CA, USA) using Taqman probe/primer sets specific for human FoxO3a, ALDH1A1, ALDH3A1, and Bcl-6. GAPDH was used as a reference for gene amplification (Applied Biosystems). Cells were stained with antibodies against CD20, CD27, CD30, immunoglobulin (Ig) $\kappa$, and $\operatorname{Ig} \lambda$ (BD Biosciences), and were analyzed with a FACS Canto II (BD Biosciences).

\section{Immunoglobulin Gene Structure}

Genomic DNA of ALDH-high and ALDH-low cell populations was obtained and used for gene rearrangement analysis according to the BIOMED-2 protocol. ${ }^{28}$ The amplified products were subject to capillary electrophoresis, and the pattern was compared between these two populations.

\section{Immunocytochemistry for FoxO3a and Ki-67}

FoxO3a and Ki-67 expression was immunocytochemically examined with anti-FoxO3a (Cell Signaling Technology, Beverly, MA, USA) and anti-Ki-67 (DAKO A/S, Glostrup, Denmark) antibodies, respectively. Cells were cytospun on poly-L-lysine-coated glass slides at $1500 \mathrm{rpm}$ for $3 \mathrm{~min}$, fixed with $10 \%$ formalin for $10 \mathrm{~min}$, and treated with $0.25 \%$ Triton X-100 solution for $10 \mathrm{~min}$. After treatment with peroxidase blocking solution (DAKO), cells were incubated with antiFoxO3a and anti-Ki-67 antibodies diluted 1:100 and treated with the ChemMate EnVision kit (DAKO). Diaminobenzidine (DAKO) was used as a chromogen. As the negative control, staining was performed in the absence of primary antibody. 


\section{Mice and Xenograft Transplantation}

Six- to 8-week-old female nonobese diabetic/severe combined immunodeficient (NOD/SCID) mice were purchased from Charles River Laboratories Japan and kept under specific pathogen-free conditions. Before xenotransplantation, the mice were deeply anesthetized. All animal experiments were performed according to the guidelines of the Osaka University Animal Center and approved by the Institutional Review Board Committee of Animal Experiments. In this study, the total number of cells was prepared at $1 \times 10^{5}$. Cells were suspended in $0.2 \mathrm{ml}$ Matrigel (BD Biosciences) and were subcutaneously injected into the flank on the left and right sides, respectively, of the same animal. Tumor volume was estimated using the formula: (width) $)^{2} \times($ length) $/ 2$, according to the report by Meyer-Siegler et al. ${ }^{29}$

\section{Knocked-Down of ALDH1A1 Expression}

For knocked-down of ALDH1A1 gene expression, cells were transfected with FlexiTube GeneSolution for ALDH1A1 (Qiagen, 4 types of siRNA were provided) or control All Star Negative Control siRNA (Qiagen). The knocked-down of ALDH1A1 gene was evaluated by qRT-PCR as described above.

\section{Cell Cycle Analysis}

The DNA contents of cells were measured by the PI staining method. Cells $\left(1 \times 10^{6}\right)$ were washed with PBS and fixed with
$70 \%$ ethanol for at least $20 \mathrm{~min}$ at $4{ }^{\circ} \mathrm{C}$. Fixed cells were rinsed twice with PBS, and re-suspended in PBS containing $0.6 \%$ Triton X-100, $0.1 \mu \mathrm{g} / \mathrm{ml}$ RNase A (Sigma) and PI, then incubated in the dark at room temperature for $20 \mathrm{~min}$. Stained cells were analyzed by a FACS Canto II (BD Biosciences) and CellQuest analysis software.

\section{Statistical Analysis}

Statistical analyses were performed using Student's $t$-tests. Data are presented as the mean \pm standard error (s.e.) of at least three experiments and $P$ values of less than 0.05 were considered to be statistically significant.

\section{RESULTS}

\section{Characteristics of ALDH-High Cells}

ALDH activity was examined using the Aldefluor assay in the HL cell lines L428 and L1236. Both cell lines contained ALDH-high cells (Figures 1a and b), though there were more in the L428 cell line than in L1236; thus, hereafter, L428 cells were the focus of our investigation. CICs are known to yield both CICs and non-CICs, whereas non-CICs do not yield any CICs. To examine whether ALDH could be used as a CIC marker for HL, ALDH-high and -low L428 cells were sorted and separately cultured. The sorted ALDH-high cells yielded both ALDH-high and -low populations, whereas ALDH-low cells yielded substantially fewer ALDH-high cells (Figures 1c and $\mathrm{d}$ ).
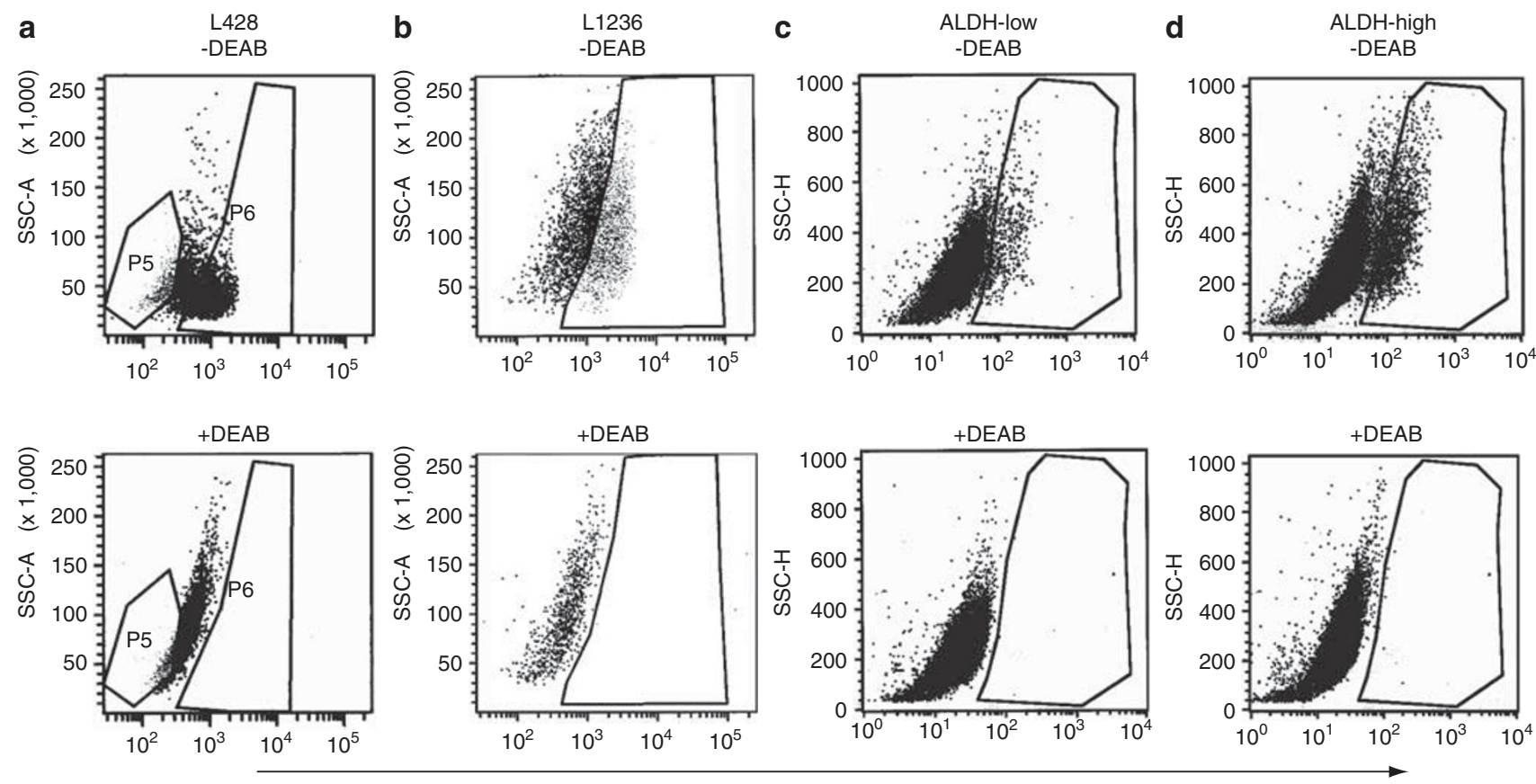

Green flourescence

Figure 1 ALDH activity in HL cells. The Aldefluor assay was performed with (a) L428 cells and (b) L1236 cells. ALDH-high (box, P6) and ALDH-low (box, P5) populations of L428 cells were sorted. The sorted cells were cultured separately for several hours and the Aldefluor assay was then repeated with both (c) ALDH-low and (d) ALDH-high (box) cells. 
a

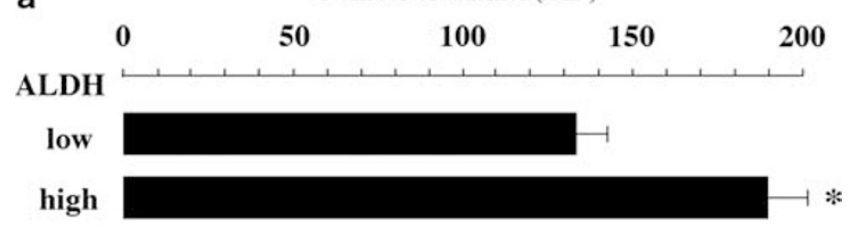

b
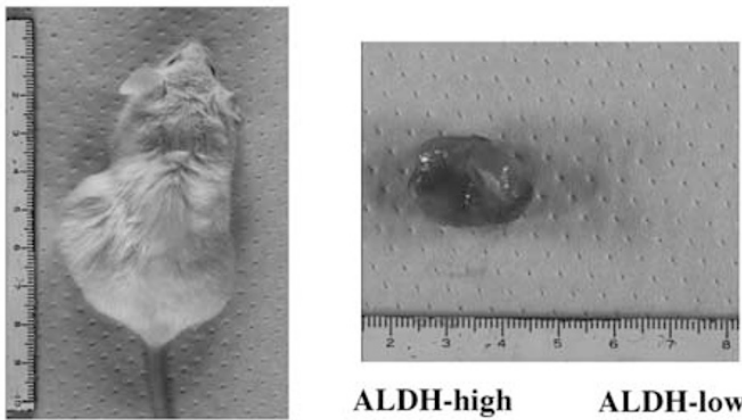

ALDH-high

ALDH-low

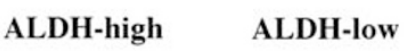

C

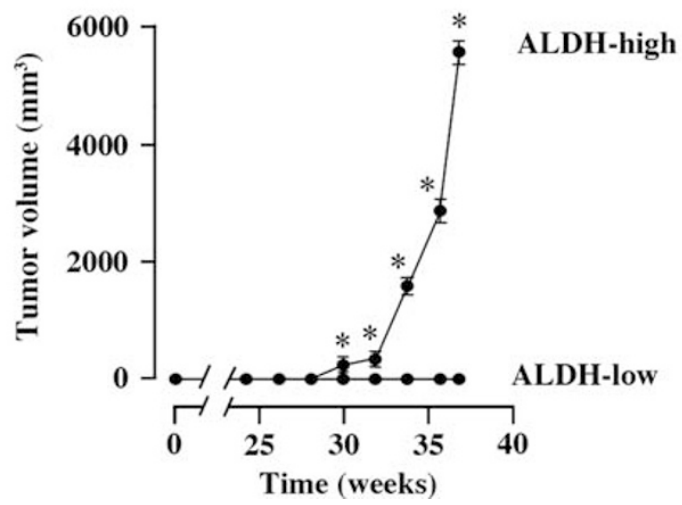

Figure 2 In vitro colony formation assay and xenotransplantation into NOD/SCID mice. (a) The number of colonies that developed during culture of ALDH-low and -high cells in methylcellulose is shown. Data are presented as the mean \pm s.e. of three experiments. ${ }^{\star} P<0.05$, Student's $t$-test. (b) Xenotransplantation of ALDH-high and -low L428 cells into NOD/SCID mice. ALDH-high and -low cells were injected into the left and right flanks, of NOD/SCID mice, respectively. A tumor mass was found at the injection site of ALDH-high cells (left), but not of ALDH-low cells (right). (c) Tumor volume is presented as the mean \pm s.e. of three experiments. ${ }^{*} P<0.05$, Student's $t$-test.

To assess their colony formation ability, the sorted ALDHhigh and -low populations were plated in methylcellulosecontaining DMEM supplemented with 15\% FCS. The number of colonies obtained from ALDH-high cells was significantly higher than that from ALDH-low cells (Figure 2a).

To examine tumorigenicity in vivo, ALDH-high and -low cells were injected into the left and right flanks, respectively, of NOD/SCID mice. At 30 weeks after the injection, a tumor mass was only found at the injection site of ALDH-high cells; tumor volumes were $112.5 \pm 8.8 \mathrm{~mm}^{2}, \quad 434.0 \pm 9.9 \mathrm{~mm}^{2}$, $1518.0 \pm 46.7 \mathrm{~mm}^{2}, 3045.0 \pm 74.2 \mathrm{~mm}^{2}$, and $5896.0 \pm 31.1 \mathrm{~mm}^{2}$ at $30,32,34,36$, and 37 weeks, respectively. No tumor was

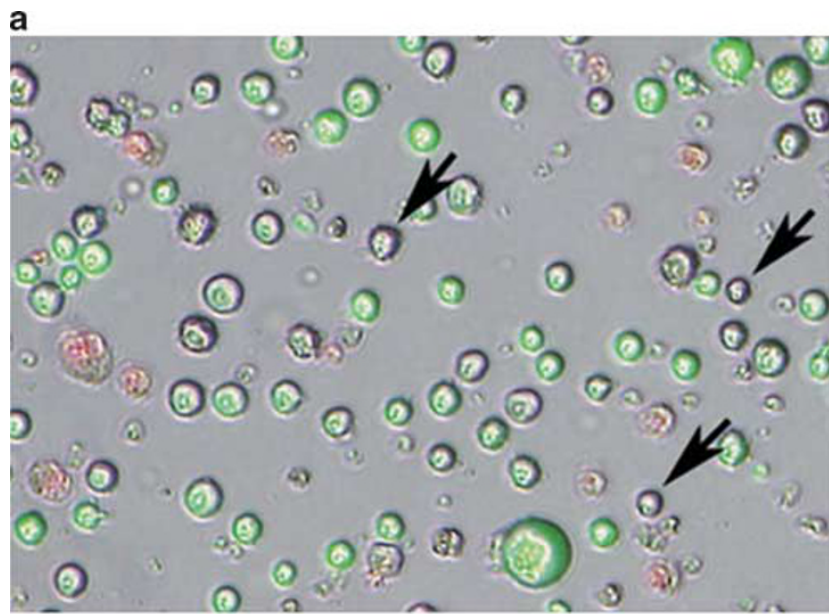

b

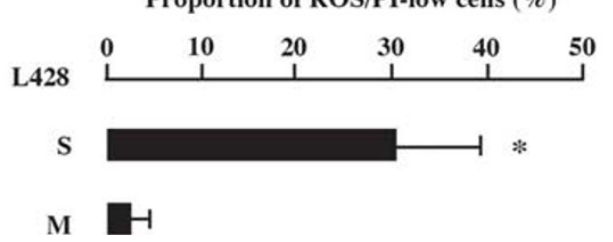

Figure 3 ROS concentration in L428 cells. (a) ROS-high cells were stained in green and dead cells are in red. Viable ROS-low cells are indicated by arrows. (b) Proportion of ROS/PI-low cells. S; single nucleus. $M$; multinucleus. Data are presented as the mean \pm s.e. of three experiments. ${ }^{\star} P<0.05$, Student's $t$-test.

found at the injection site of ALDH-low cells throughout the observation period (Figures $2 \mathrm{~b}$ and $\mathrm{c}$ ).

To further examine the tumorigenic potential of ALDH-high cells, the knock-down of ALDH1A1 expression was performed. Although ALDH1A1 expression was downregulated in L428 cells (Supplementary Figure 1A), the ALDH-high population was not completely absent (Supplementary Figure 1B). This suggested that ALDH activity of L428 cells was attributable not only to ALDH1A1, but also to other isoforms of ALDH. Therefore, ALDH activity was not downregulated by conventional knock-down of the ALDH1A1 gene alone.

Characteristics of the ROS-Low Population of L428 Cells In addition to high ALDH activity, ROS-degradation ability is one of the characteristics of CICs. Previously, we demonstrated that the ROS contents in a small portion of L1236 cells persisted at a low level after culture. ${ }^{26}$ This ROS-degrading population of L1236 cells was tumorigenic and small in cell size. To examine whether this observation was applicable to L428, cells were stained with $\mathrm{CM}-\mathrm{H}_{2} \mathrm{DCFDA}$, and cells with high levels of ROS fluoresced green. When stained with CM- $\mathrm{H}_{2} \mathrm{DCFDA}$, a proportion of $\mathrm{L} 428 \mathrm{~S}$ cells had no or faint green fluorescence, indicating that an ROS-degrading population was also present among L428 cells (Figures 3a and $b$ ). 


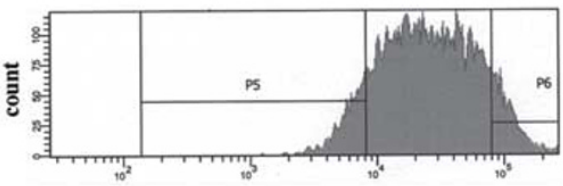

Green Fluorescence
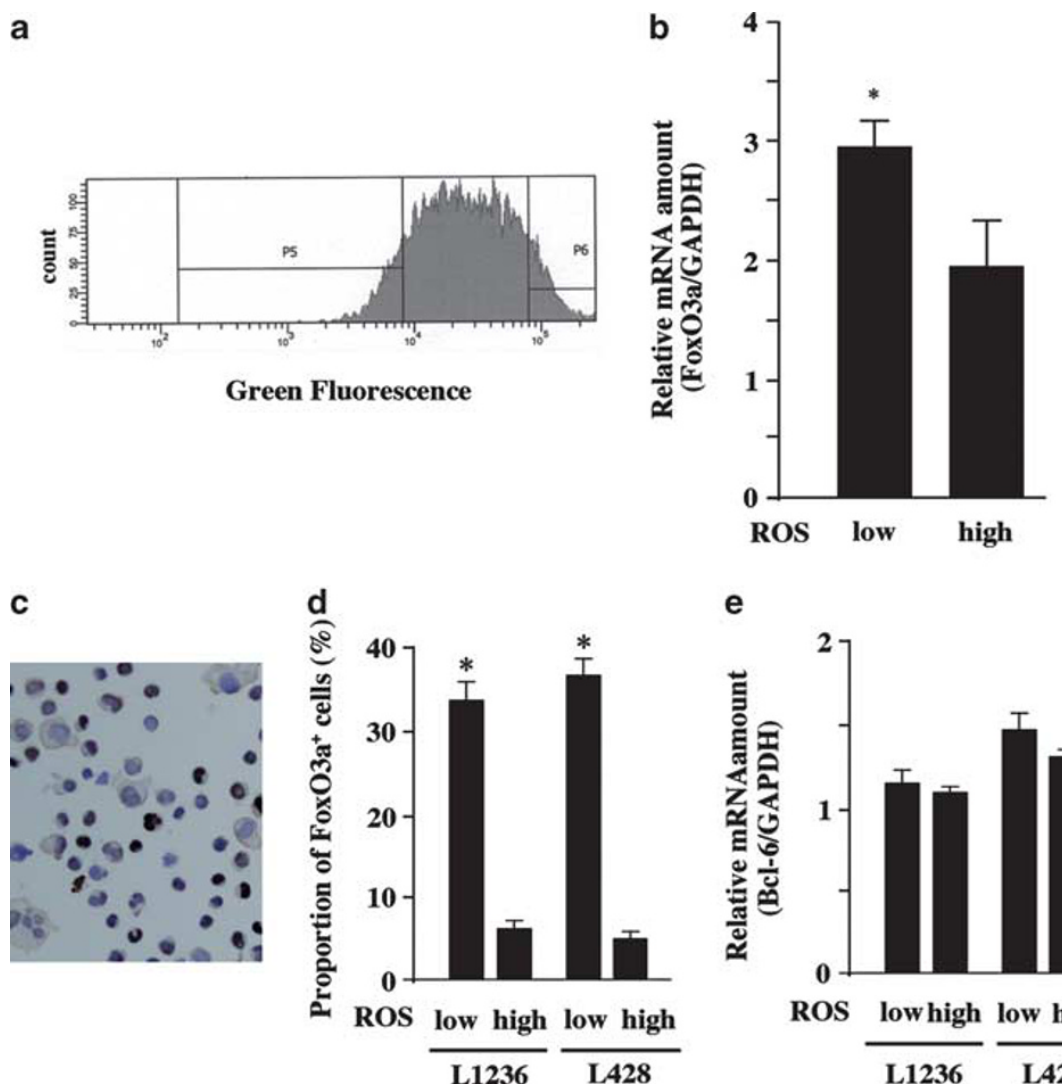

e

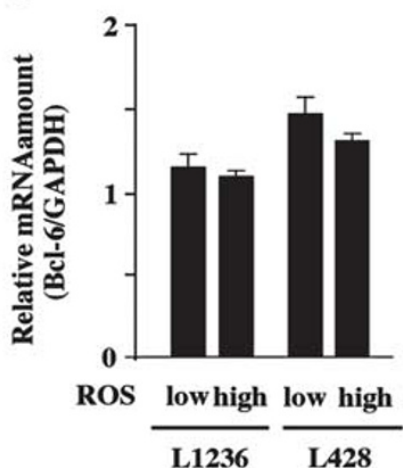

Figure 4 Expression of FoxO3a in ROS-low and ROS-high L428 cells. (a) Flow cytometric analysis of CM-H ${ }_{2}$ DCFDA staining. ROS-low and ROS-high populations were gated. (b) Real-time qRT-PCR for FoxO3a expression in ROS-low and -high cells. (c) Immunocytochemistry of FoxO3a in L428 cells ( $\times 400$ ). (d) Proportion of FoxO3a-positive cells in ROS-low and -high cell populations. (e) Real-time qRT-PCR for Bcl-6 expression in ROS-low and -high cells. Data are presented as the mean \pm s.e. of three experiments. ${ }^{\star} P<0.05$, Student's $t$-test.

It is known that ROS and FoxO3a levels are inversely correlated. As was previously reported in L1236 cells, sorted ROS-low L428 cells (Figure 4a) expressed higher levels of FoxO3a mRNA than ROS-high cells (Figure 4b). Immunocytochemically, FoxO3a expression was mostly confined to a portion of $\mathrm{S}$ cells (Figures $4 \mathrm{c}$ and $\mathrm{d}$ ). These findings indicated that some $\mathrm{S}$ cells with in the L428 cell population expressed a higher level of FoxO3a and expelled ROS efficiently.

Bcl-6 is reported to suppress ROS production in B-non $\mathrm{HL}^{30}$ To examine whether this is the case in HL, Bcl-6 expression levels were examined in ROS-high and -low L1236 and L428 cells. No significant difference in Bcl-6 expression was detected between these two populations, indicating that Bcl-6 was not involved in the regulation of ROS production, at least in L1236 and L428 cells (Figure 4e).

To assess the colony formation ability of ROS-low and -high cells, the two cell populations were plated separately. The number of colonies obtained from ROS-low cells was significantly higher than that from ROS-high cells (Figure 5a). To examine tumorigenicity in vivo, ROS-low and -high cells were injected into the left and right flanks of NOD/SCID mice, respectively. At 12 weeks post-injection, a tumor mass was only found at the injection site of
ROS-low cells; tumor volumes were $210.0 \pm 9.9 \mathrm{~mm}^{2}$, $1894.5 \pm 92.3 \mathrm{~mm}^{2}$, and $4992.0 \pm 135.8 \mathrm{~mm}^{2}$ at days 98,119 , and 133, respectively. Tumors derived from ROS-low cells consisted of both $\mathrm{S}$ and $\mathrm{M}$ cells. No tumor was found at the injection site of ROS-high cells throughout the observation period (Figures $5 \mathrm{~b}$ and $\mathrm{c}$ ).

\section{Immunophenotype and Immunoglobulin Gene Structure of ALDH-High and-Low Populations}

The immunophenotypes of ALDH-high and ALDH-low populations of L428 cells were examined. The ALDH-high population contained a greater number of CD30-negative cells than the ALDH-low population (Figure 6a). A small number of CD20-positive cells was found in the ALDH-high, but not in the ALDH-low, population (Figure 6a). The proportion of CD27-positive cells in the ALDH-high population was comparable to that in the ALDH-low population. Restricted expression of the Ig light chain $(\operatorname{Ig} \lambda)$ was found in the ALDH-high population (Figure 6a), but not in the ALDH-low population.

Next, the Ig gene sequence was compared between ALDHhigh and -low populations. The capillary electrophoretic 

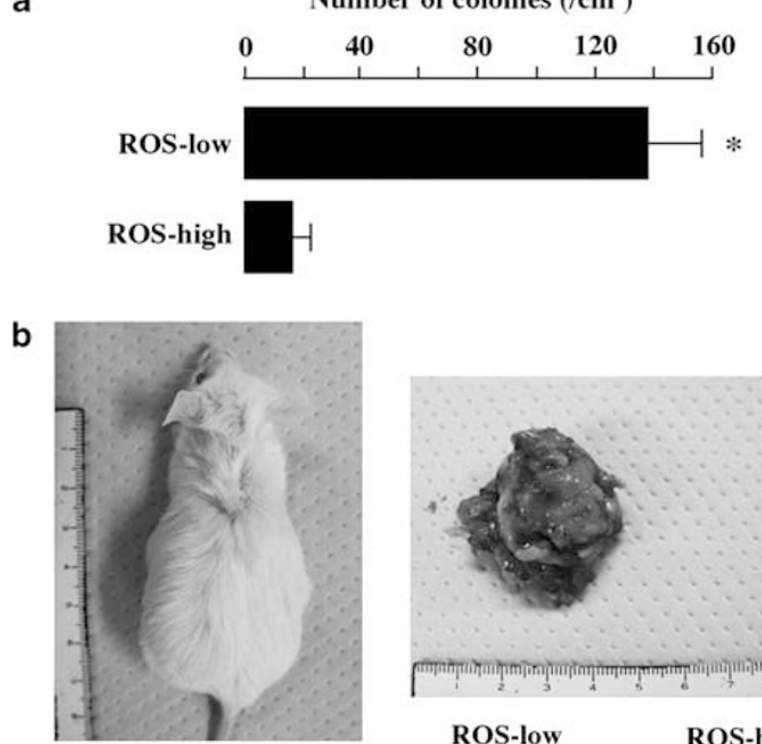

$$
\text { ROS-low ROS-high }
$$

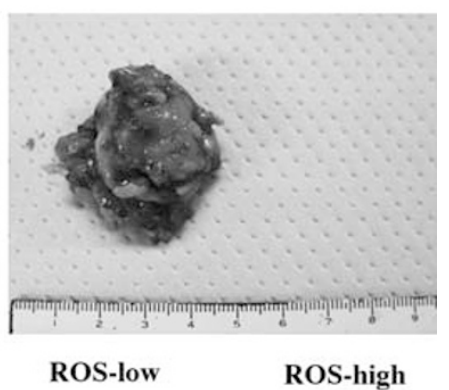

C

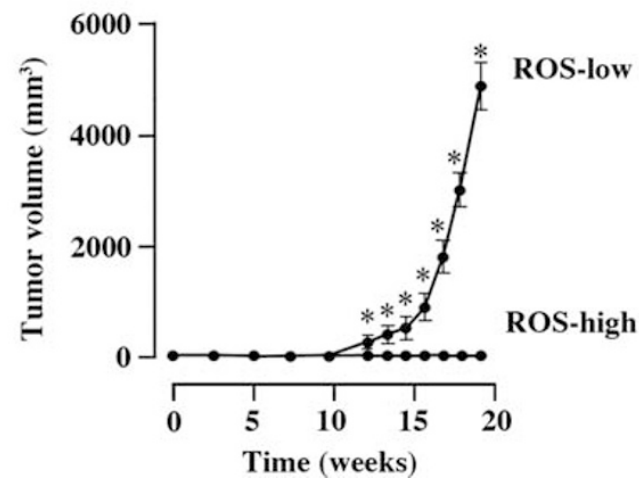

Figure 5 In vitro colony formation assay and xenotransplantation into NOD/SCID mice. (a) In vitro colony formation assay. The number of colonies that developed during culture of ROS-low and -high cells in methylcellulose is shown. (b) Xenotransplantation of ROS-low and -high L428 cells into NOD/SCID mice. ROS-low and -high cells were injected into the left and right flanks of NOD/SCID mice, respectively. A tumor mass was found at the injection site of ROS-low cells, but not of ROS-high cells. Resected tumor at the injection site of ROS-low cells (left). No tumor was found at the injection site of ROS-high cells (right). (c) Tumor volumes derived from mice injected with ROS-low and -high cells. The data are presented as the mean \pm s.e. of three experiments. ${ }^{*} P<0.05$, Student's $t$-test.

profiles of $\kappa$ chain amplification were comparable between these two populations (Figure 6b).

\section{Relationship between ROS-Low and ALDH-High Populations}

The correlation between ROS-degradation and ALDH activity was examined. In both L1236 and L428 cells, the level of ALDH1A1 expression was significantly higher in ROS-low than in ROS-high cells (Figures 7a and b). The expression of another isoform of ALDH, ALDH3A1, was also examined, a
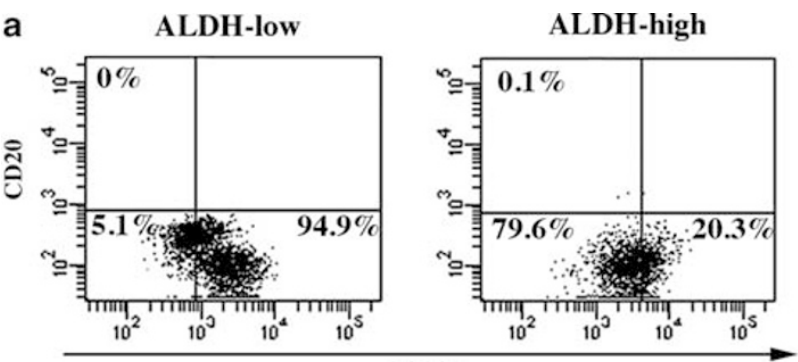

CD30
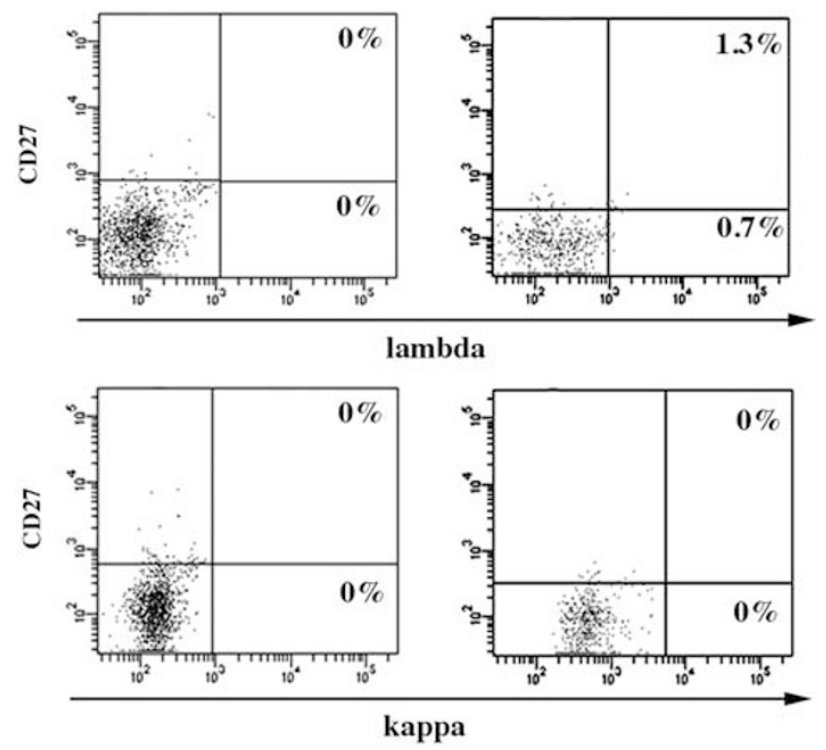

b ALDH-high

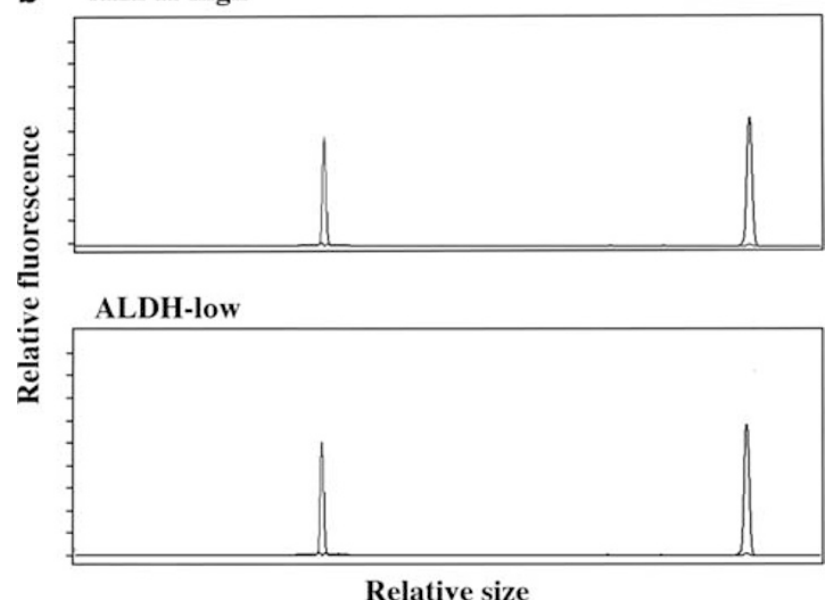

Figure 6 Immunophenotype and immunoglobulin gene sequence of ALDH-high and ALDH-low populations of L428 cells. (a) The surface expression levels of CD20, CD30, CD27, $\lambda$ chain, and $\kappa$ chain were examined. (b) Capillary electrophoretic profiles of amplified products from immunoglobulin $\kappa$ chain, following the BIOMED-2 protocol.

but no significant difference was detected between ROS-low and -high cells (Supplementary Figure 2). Immunocytochemically, the proportion of FoxO3a-positive cells among L1236 and L428 cells was higher in ALDH-high than in 

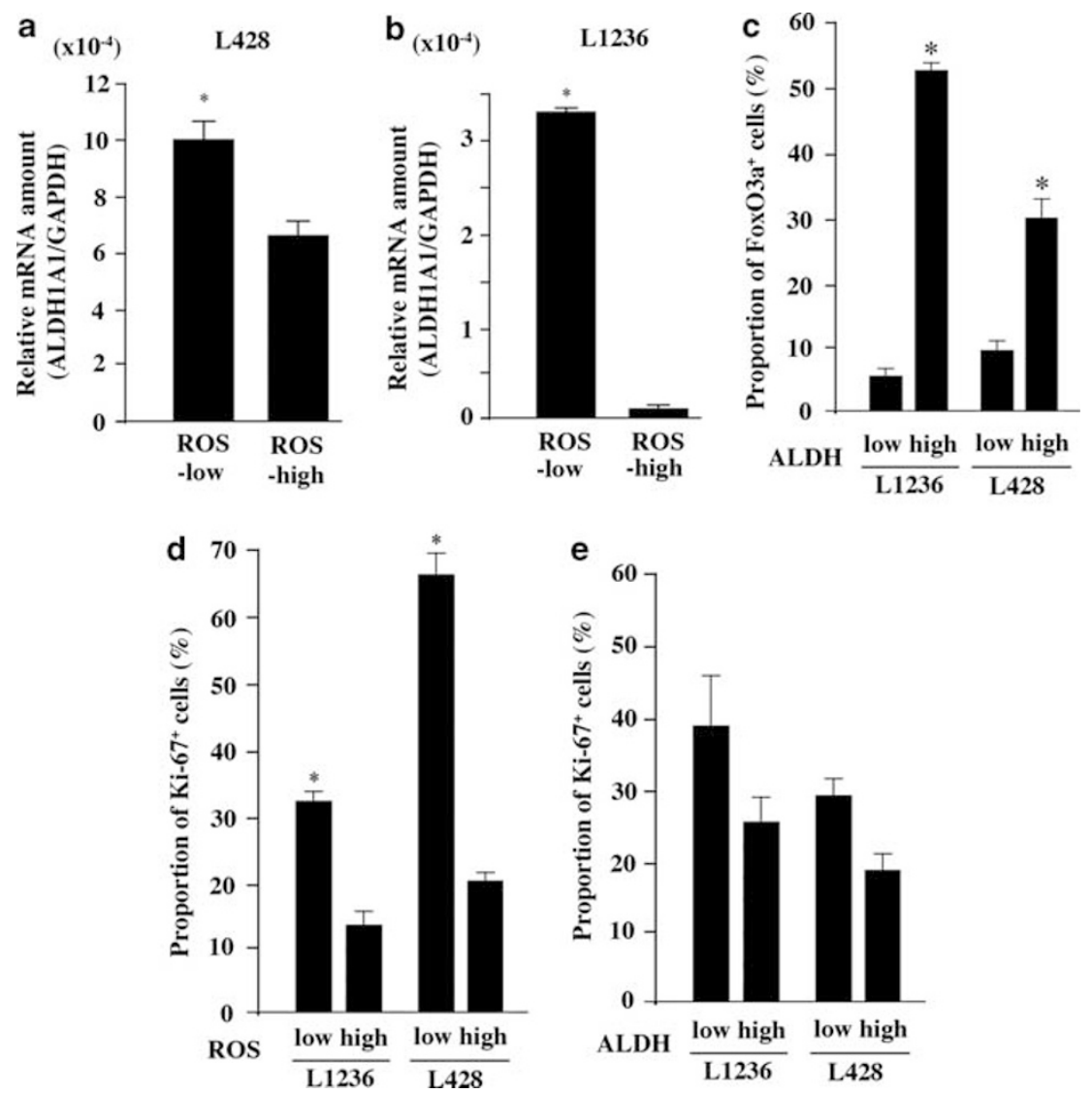

Figure 7 Real-time qRT-PCR of ALDH1A1 expression in ROS-low and ROS-high HL cells. ALDH1A1 expression was determined in (a) L428 and (b) L1236 cell lines. (c) Proportion of FoxO3a-positive cells in the ALDH-low and -high populations. (d, e) Proportion of Ki-67 positive cells in ROS-low and -high (d), or ALDH-low and -high cells (e). Data are presented as the mean \pm s.e. of three experiments. ${ }^{\star} P<0.05$, Student's $t$-test.

ALDH-low cells, as in the case of ROS-low cells shown in Figure 4d (Figure 7c). In terms of proliferative activity, although the proportion of Ki-67-positive cells was significantly higher in ROS-low than in ROS-high populations, the proportion of Ki-67-positive cells was not significantly different between ALDH-high and -low cells (Figures 7d and e).

To examine cell cycle status, the proportions of G0/G1, $\mathrm{S}, \mathrm{G} 2 / \mathrm{M}$, and sub-G0 cells were evaluated by flow cytometry. Immediately after sorting, ROS-low cells had more cells in the G0/G1 phase than ROS-high cells (Figures $8 \mathrm{a}$ and b). In contrast, the proportion of cells in the G0/G1 phase was not significantly different between ALDH-high and -low cells (Figures $8 \mathrm{c}$ and $\mathrm{d}$ ).

The change in cell cycle status during culture of sorted cells was also examined. Twenty-four hours after the start of culture of sorted ROS-low cells, the proportion of G0/G1 phase cells decreased, whereas those in the G2/M phase increased (Figure 8a). The number of ROS-high cells in the G0/G1 phase did not change, whereas an increase of sub-G0 phase cells and the appearance of apoptosis were found (Figure $8 \mathrm{~b}$ ). Increased numbers of sub-G0 cells were not a

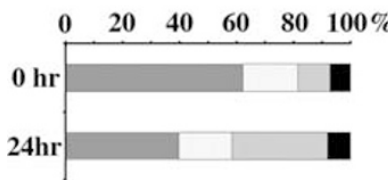

C
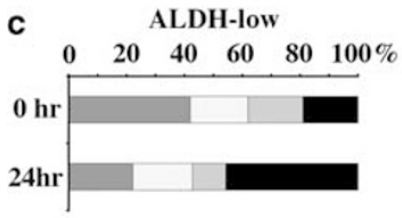

G0/G1 b

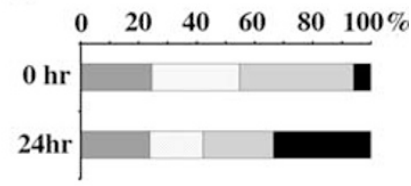

d
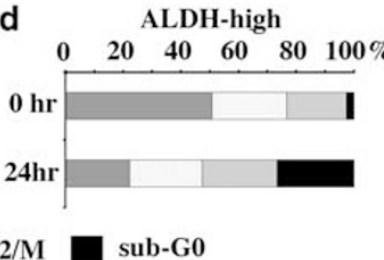

Figure 8 Cell cycle analysis. The cell cycle statuses of (a) ROS-low, (b) ROS-high, (c) ALDH-low, and (d) ALDH-high cells were determined after $24 \mathrm{~h}$ of culture.

detected in ROS-low cells. The proportion of sub-G0 cells increased in ALDH-low cells (Figure 8c). Approximately half of the ALDH-low cells were in the sub-G0 phase, which was significantly more than that found in ALDH-high cells (Figure 8d). 


\section{DISCUSSION}

Historically, the origin of neoplastic cells, e.g., RS cells, in HL has been of great interest. Recent studies analyzing immunoglobulin gene rearrangement and the fusion patterns of terminal repeats in Epstein-Barr virus (EBV) in microdissected single cells from HL lesions revealed the monoclonality and neoplastic B cell nature of RS and Hodgkin cells. ${ }^{23,24}$ Our recent study using the HL cell lines L1236 and L428 revealed that a portion of large cells with a single nucleus ( $\mathrm{S}$ cells) that resemble Hodgkin cells have tumorigenic potential. $^{26}$ This tumorigenic population expelled ROS efficiently and maintained ROS at low levels. Since ROSdegradation is characteristic of CICs, the tumorigenic population of $\mathrm{S}$ cells was suggested as a candidate CIC of HL.

In addition to ROS-degradation, cells with high ALDH activity are reported to possess CIC characteristics; the ALDH-high population is tumorigenic and resistant to chemotherapy in cancers of the colon, breast, lung, pancreas, bladder, prostate, and ovary. ${ }^{10-20}$ In the present study, the ALDH-high population of HL was examined. ALDH-high cells efficiently formed colonies and yielded both ALDH-high and -low populations. In contrast, ALDH-low cells rarely yielded ALDH-high cells. These findings were in agreement with the study by Jones $e a^{27}$ that indicated the circulating B cell population with high ALDH expression in HL patients was tumorigenic. That same study also reported that tumorigenic potential was detected in the CD30-negative, CD20-positive, and Ig light chain-expressing population of HL. Consistent with this, the ALDH-high population contained CD30-negative, CD20-positive, and $\lambda$ chain-expressing cells more abundantly than the ALDH-low population. ALDH-high cells, in particular less differentiated cells, may possess characteristics of CICs in HL, similar to that seen in cancers of other organs.

CICs are known to expel ROS efficiently and possess high ALDH activity. ${ }^{9-20}$ However, to our knowledge, it was unclear whether ROS-low cells and ALDH-high cells merely shared $\mathrm{CIC}$ characteristics or belonged to the same population of cells. Therefore, this study examined ALDH activity in ROS-low and -high cells. ALDH expression levels were significantly higher in ROS-low than in -high cells. FoxO3a activates various ROSexpelling enzymes, and its expression level is reported to be high in ROS-low cells. ${ }^{26,31,32}$ The present study revealed that the proportion of FoxO3a expressing cells was higher in the ALDH-high than in the ALDH-low population. These findings suggest that the ALDH-high and ROS-low populations appear to be identical, or, at least, overlapped.

The proportion of Ki-67-positive cells was significantly higher in ROS-low than -high cells, whereas ALDH-high and -low populations contained similar proportions of Ki-67positive cells. Upon cell cycle analysis, the proportion of cells in the G0/G1 phase was higher in ROS-low than -high cells. In contrast, no difference in the proportion of cells in the G0/G1 phase was found between ALDH-high and -low cells. These findings indicated that the ROS-low population was different from ALDH-high cells with regard to cell cycle status. As CICs are known to be relatively quiescent in several tumors, ${ }^{16,33,34}$ ROS-low cells are more likely to be CIC than ALDH-high populations. Further studies on CICs in various types of malignancies, other than HL, will provide insight on the precise relationship between ROS-expelling and high ALDH activities.

Moreb et $a l^{35}$ recently reported that the knock-down of $A L D H 1 A 1$ and $A L D H 3 A 1$ resulted in decreased proliferative activity of lung adenocarcinoma cell lines. Since the level of ALDH did not affect the proliferative activity of Hodgkin lymphoma cell lines in the present study, the effect of ALDH may differ among cancer cell types. In fact, high ALDH activity is a poor prognostic indicator for breast, prostate, bladder, and uterine cancers, but a favorable indicator for ovary cancer. ${ }^{36,37}$ Further studies are necessary to elucidate the mechanism of ALDH function in cancer cells.

The ROS-low population in a quiescent state efficiently formed colonies in vitro, although rapid proliferation of cells might be necessary for colony formation. When compared to the cell cycle status just after sorting, the ROS-low population showed a dramatic increase in the number of G2/M phase cells after $24 \mathrm{~h}$ of culture. In contrast, the proportion of G2/M phase cells decreased and apoptosis occurred in the ROS-high population. The lower number of colonies found during the culture of ROS-high cells compared to -low cells might be due to low proliferative potential and vulnerability to apoptosis. ALDH-high cells had a similar ability for efficient colony formation as ROS-low cells. After $24 \mathrm{~h}$ in culture, the proportion of apoptotic cells was lower in the ALDH-high than in the ALDH-low population. Anti-apoptotic potential may be attributable to the ability for efficient colony formation in the ALDH-high population.

Taken together, ALDH-high and ROS-low populations may be candidate CICs; however, they have distinct cell cycle profiles, indicating that CICs may be comprised of cells with heterogeneous characteristics.

Supplementary Information accompanies the paper on the Laboratory Investigation website (http://www.laboratoryinvestigation.org)

\section{ACKNOWLEDGEMENTS}

The authors thank Ms. Megumi Nihei-Sugano, Ms. Etsuko Maeno, and Ms. Takako Sawamura for their technical assistance. This work was supported by grants from the Ministry of Education, Culture, Sports, Science and Technology and from the Osaka Cancer Research Foundation. This work was supported by Grants from the Ministry of Education, Culture, Sports, Science and Technology, Japan (\#23590425, \#23790397), and from the Ministry of Health, Labour and Welfare, Japan (\#KH22Q151b).

\section{DISCLOSURE/CONFLICT OF INTEREST}

The authors declare no conflict of interest.

1. Bonnet D, Dick JE. Human acute myeloid leukemia is organized as a hierarchy that originates from a primitive hematopoietic cell. Nat Med 1997;3:730-737.

2. Lessard J, Sauvageau G. Bmi-1 determined the proliferative capacity of normal and leukaemic stem cells. Nature 2003;423:255-260. 
3. Al-Hajj M, Wicha MS, Benito-Hernandez A, et al. Prospective identification of tumorigenic breast cancer cells. Proc Natl Acad Sci USA 2003:100:3983-3988.

4. Singh SK, Hawkins C, Clarke ID, et al. Identification of human brain tumour initiating cells. Nature 2004;432:396-401.

5. Reya T, Morrison SJ, Clarke MF, et al. Stem cells, cancer, and cancer stem cells. Nature 2001;414:105-111.

6. O'Brien CA, Pollett A, Gallinger S, et al. A human colon cancer cell capable of initiating tumor growth in immunodeficient mice. Nature 2007;445:106-110.

7. Ricci-Vitiani L, Lombardi DG, Pilozzi E, et al. Identification and expansion of human colon-cancer-initiating cells. Nature 2007;445:111-115.

8. Kondo T, Setoguchi T, Taga T. Persistence of a small subppulation of cancer stem-like cells in the C6 glioma cell line. Proc Natl Acad Sci USA 2004;101:781-786.

9. Diehn M, Cho RW, Lobo NA, et al. Association of reactive oxygen species levels and radioresistance in cancer stem cells. Nature 2009;458:780-783.

10. Ginestier $\mathrm{C}$, Hur MH, Charafe-Jauffret $\mathrm{E}$, et al. ALDH1 is a marker of normal and malignant human mammary stem cells and a predictor of poor clinical outcome. Cell Stem Cell 2007;1:555-567.

11. Chute JP, Muramoto GG, Whitesides J, et al. Inhibition of aldehyde dehydrogenase and retinoid signaling induces the expansion of human hematopoietic stem cells. Proc Natl Acad Sci USA 2006; 103:11707-11712.

12. Liu S, Ginestier C, Charafe-Jauffret $\mathrm{E}$, et al. BRCA1 regulates human mammary stem progenitor cell fate. Proc Natl Acad Sci USA 2008; 105:1680-1685.

13. Ibarra I, Erlich $\mathrm{Y}$, Muthuswamy SK, et al. A role for microRNAs in maintenance of mouse mammary epithelial progenitor cells. Genes Dev 2007;21:3238-3243.

14. Dave B, Chang J. Treatment resistance in stem cells and breast cancer. J Mammary Gland Biol Neoplasia 2009;14:79-82.

15. Jiang F, Qiu Q, Khanna A, et al. Aldehyde dehydrogenase 1 is a tumor stem cell-associated marker in lung cancer. Mol Cancer Res 2009;7:330-338.

16. Matsui W, Wang Q, Barber JP, et al. Clonogenic multiple myeloma progenitors, stem cell properties, and drug resistance. Cancer Res 2008;68:190-197.

17. Pearce DJ, Taussig D, Simpson C, et al. Characterization of cells with a high aldehyde dehydrogenase activity from cord blood and acute myeloid leukemia samples. Stem Cells 2005;23:752-760.

18. Rasheed ZA, Yang J, Wang Q, et al. Prognostic significance of tumorigenic cells with mesenchymal features in pancreatic adenocarcinoma. J Natl Cancer Inst 2010;102:340-351.

19. Su Y, Qiu Q, Zhang X, et al. Aldehyde dehydrogenase 1 A1-positive cell population is enriched in tumor-initiating cells and associated with progression of bladder cancer. Cancer Epidemiol Biomarkers Prev 2010;19:327-337.

20. Li T, Su Y, Mei Y, et al. ALDH1A1 is a marker for malignant prostate stem cells and predictor of prostate cancer patients' outcome. Lab Invest 2010;90:234-244.

21. Kastan MB, Schlaffer E, Russo JE, et al. Direct demonstration of elevated aldehyde dehydrogenase in human hematopoietic progenitor cell. Blood 1990;75:1947-1950.
22. Corti $\mathrm{S}$, Locatelli $\mathrm{F}$, Papadimitriou $\mathrm{D}$, et al. Identification of a primitive brain-derived neural stem cell population based on aldehyde dehydrogenase activity. Stem Cells 2006;24:975-985.

23. Kanzler H, Kuppers R, Hansmann ML, et al. Hodgkin and ReedSternberg cells in Hodgkin's disease represent the outgrowth of a dominant tumor clone derived from (crippled) germinal center B cells. J Exp Med 1996;184:1495-1505.

24. Marofioti T, Hummel M, Foss HD, et al. Hodgkin and reed-sternberg cells represent an expansion of single clone originating from a germinal center B-cell with functional immunoglobulin gene rearrangements but defective immunoglobulin transcription. Blood 2000;95:1443-1450.

25. Hsu SM, Zhao X, Chakraborty S, et al. Reed-Sternberg cells in Hodgkin's cell lines HDLM, L-428, and $\mathrm{KM}-\mathrm{H} 2$ are not actively replicating: lack of bromodeoxyuridine uptake by multinuclear cells in culture. Blood 1988;71:1382-1389.

26. Ikeda J, Mamat S, Tian T, et al. Tumorigenic potential of mononucleated small cells of Hodgkin lymphoma cell lines. Am J Pathol 2010;177:3081-3088.

27. Jones RJ, Gocke CD, Kasamon YL, et al. Circulating clonotypic B cells in classic Hodgkin lymphoma. Blood 2009;113:5920-5926.

28. vanDongen JJM, Langerak AW, Bruggemann $M$, et al. Design and standardization of PCR primers and protocols for detection of clonal immunoglobulin and T-cell receptor gene recombinations in suspect lymphoproliferations: report of the BIOMED-2 concerted action BMH4CT98-3936. Leukemia 2003;17:2257-2317.

29. Meyer-Siegler KL, Iczknowski KA, Leng $\mathrm{L}$, et al. Inhibition of macrophage migration inhibitory factor or its receptor (CD74) attenuates growth and invasion of DU-145 prostate cancer cells. J Immunol 2006;177:8730-8739.

30. Kurosu $\mathrm{T}$, Fukuda $\mathrm{T}$, Miki $\mathrm{T}$, et al. BCL6 overexpression prevents increase in reactive oxygen species and inhibits apoptosis induced by chemotherapeutic reagents in B-cell lymphoma cells. Oncogene 2003;22:4459-4468.

31. Tothova Z, Kollipara R, Huntly BJ, et al. FoxOs are critical mediators of hematopoietic stem cell resistance to physiologic oxidative stress. Cell 2007;128:325-339.

32. Miyamoto K, Araki KY, Naka K, et al. FoxO3a is essential for maintenance of hematopoietic stem cell pool. Cell Stem Cell 2007;1: 101-112.

33. Guan Y, Gerhard B, Hogge DE. Detection, isolation and stimulation of quiescent primitive leukemic progenitor cells from patients with acute myeloid leukemia (AML). Blood 2003;8:3142-3149.

34. Holyoake $T$, Jiang $X$, Eaves $C$, et al. Isolation of a highly quiescent subpopulation of primitive leukemic cells in chronic myeloid leukemia. Blood 1999:94:2056-2064.

35. Moreb JS, Barker HV, Chang LJ, et al. ALDH isozymes downregulation affects cell growth, cell mobility and gene expression in lung cancer cells. Mol Cancer 2008;7:87.

36. Ma I, Allan AL. The role of human aldehyde dehydrogenase in normal and cancer stem cells. Stem Cell Rev 2011;7:292-306.

37. Rahadiani $\mathrm{N}$, Ikeda $\mathrm{H}$, Mamat $\mathrm{S}$, et al. Expression of aldehyde dehydrogenase 1 (ALDH1) in endometrioid adenocarcinoma and its clinical implications. Cancer Sci 2011;102:903-908. 